Physics

\section{Low-dimensional electron movement in a magnetic field}

\author{
from Paul Chaikin
}

ELECTRONS in two dimensions in a perpendicular magnetic field move in circular orbits. Quantization of these orbits is responsible for the quantum Hall effect as well as a wealth of oscillatory phenomena in metals. But electrons in one dimension in a magnetic field cannot form such orbits. The discovery of a series of magnetic field-induced phase transitions, oscillations and behaviour like the quantum Hall effect in Bechgaard salts, which act as quasi-one-dimensional organic conductors, has stimulated a flurry of recent experimental $^{1-5}$ and theoretical ${ }^{6-9}$ work and the discovery of a new form of instability in a two-dimensional system ${ }^{10.11}$, as well as many unanswered questions.

The crystal potential has two interesting effects on the states of a two-dimensional system in the field. If the potential is reasonably isotropic (as in a square lattice) the electron orbits are distorted but closed and periodic. The wavefunctions must satisfy both orbital quantization and crystalline symmetry, conditions which are usually incommensurate. The result is a complex hierarchical self-similar energy spectrum with gaps on all energy scales ${ }^{12.13}$ (Fig. 1). If the potential is highly anisotropic the motion of the electron may form a zigzag pattern which goes off in the easiest direction and never closes - an open orbit (Fig. 2).

The Bechgaard salts provide a unique system for the study of an interacting twodimensional electron gas in a magnetic field. They have the formula (TMTSF) $X$ where $X$ is $\mathrm{PF}_{6}, \mathrm{ClO}_{4}, \mathrm{ReO}_{4}$ and so on ${ }^{1-5}$, and are variously regarded as quasi-oneor quasi-two-dimensional. They are better known as the first organic superconductors, but they can also have insulating spin-density wave (SDW) ground states depending on the pressure and on the anion $X$. The band structure has been calculated and measured: the anisotropy in bandwidths in the highly conducting plane

\footnotetext{
1. Kwak, J.F. et al. Phys. Rev. Lett. 46, 1296 (1981).

Schwenk, H. et al. Phys. Rev. Lett. 56, 667 (1986).

3. Kwak, J.F. et al. Phys. Rev. Lett. 56. 972 (1986).

4. Pesty, F. et al. Phys. Rev. Lett. 55, 2495 (1985)

Naughton M J et al Phys Rev Lett 55, $969(1985)$

. Montambaux, G. et al. Phys. Rev. Lett. 55, 2078 (1985).

Yamaji, K. et al. Phys. Soc., Japan 54, 1034 (1985).

Virosztek, A. et al. Phys, Rev. A (submitted)

Azbel, M. Ya et al.Phys. Rev. A (submitted).

10. Gorkov, L.P. \& Lebed, A.G. J. Phys. Lett., Paris, 45 L433 (1984).

11. Chaikin. P.M. Phys. Rev. B31. $4770(1985)$

12. Azbel, M. Ya Zh. Eksp. Teor. Fiz, 46, 939 (1964) [Sov Phys. JETP 19. 634 (1964)]

13. Hofstadter. D. R. Phys. Rev. B14. 2239 (1976).

14. Thouless, D. et al. Phys. Rev. Lett. 49. 405 (1982).

15. Ribault, M. Molec. Cryst. Liq. Cryst. 119, 91 (1985).

16. Ribault. M. et al. J. Phys. Lett. Paris 44. L953 (1983)

17. Chaikin, P.M. et al Phvs. Rev. Lett. 51, 2333 (1983).
}

is about 10:1, whereas between the planes the bandwith is smaller by a factor of 30 .

It is well known that one-dimensional metals are unstable against a Peierl's distortion or SDW which leads to an insulating ground state. For the Bechgaard salts under consideration the band-

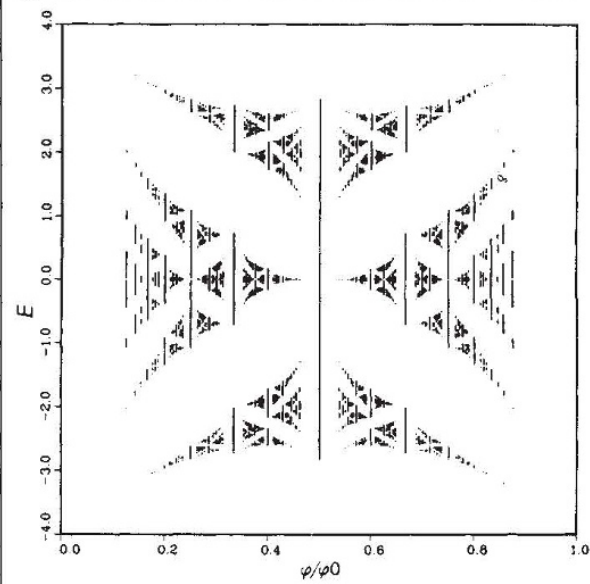

Fig. 1 Energy spectrum of an electron in a square tight binding model in the presence of a magnetic field measured in flux quanta per unit cell $\left(\phi / \phi_{0}\right)$. Dark regions, allowed states.

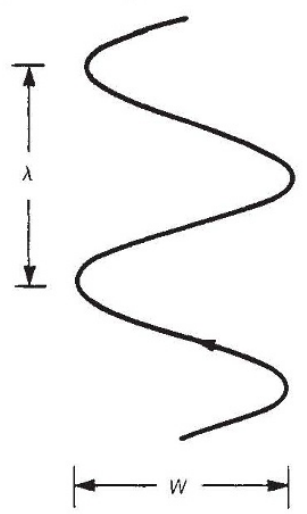

Fig. 2 Electron motion in a magnetic field for an open orbit. $w$ and $\lambda$ are proportional to the reciprocal of the magnetic field.

width in the second most conducting direction is large, the systems are effectively two-dimensional and they remain metallic to low temperatures. But the anisotropy is sufficient to make all the orbits open at the Fermi surface. In the presence of a magnetic field the electrons undergo the motion illustrated in Fig. 2. As the magnetic field increases the width $w$ of the motion along the $x$ direction and the period $\lambda$ along the $y$ direction decrease. The effect is to make the electrons more one-dimensional and thus increasingly susceptible to an SDW transition. Strictly speaking, the electron motion and the spectrum of the open-orbit electrons in a magnetic field is always one-dimensional (limited excursions along $x$ and infinite along $y$ ). Thus, two-dimensional open-orbit metals cannot exist at zero temperature. An infinitesimal magnetic field will drive them to an insulating Peierl's or SDW state . $^{12.13}$.

The basic instability leading to the fieldinduced transition is therefore well established. There are several quasi-classical models that seek to explain the series of phase transitions which allow the wavevector of the SDW distortion to change both continuously and discontinuously to take advantage of the magnetic quantization of carrier pockets which result from the distortion ${ }^{6-9}$.

In the usual treatment of the onedimensional instability a single length is considered - the reciprocal of the Fermi momentum $\left(P_{\mathrm{f}}\right)$ - and this sets the wavelength of the distortion at $\mathrm{h} / 2 P_{\mathrm{r}}$. In the field-induced phase there at least three lengths, $\mathrm{h} / 2 P_{\mathrm{f}}$, the magnetic length $\lambda$ (Fig. 2), which varies reciprocally with the magnetic field, and the lattice period. In general the lengths are incommensurate with one another. The distortion wavevector results from the competition of the interactions represented by these lengths. The spectrum resulting from incommensurate potentials is often quite complex, as illustrated for a particular case in Fig. 1.

One might expect that the existence of the small gaps in the spectrum are inconsequential, as they are usually smeared out by finite temperature or scattering. The gaps have a profound effect on the Hall conductance, with the most dramatic effects involving large changes in magnitude and sign of the Hall conductance resulting from the smallest gaps ${ }^{1+}$. More perfect crystals show an increasing number of field-induced transitions with abrupt sign changes in the Hall voltage ${ }^{15}$, suggesting that in these salts an incommensurate spectrum is involved ${ }^{3}$. The existence of oscillations with a different magnetic frequency at high fields also points to a complex spectrum ${ }^{2}$.

There are still basic questions left to be answered. The eventual state at high field should be insulating, but so far only semimetallic behaviour has been observed. And what of the quantum Hall effect? Hall measurements indicate steplike structure, but it is temperature dependent and has other dissimilarities ${ }^{15-17}$. The interplane bandwidth is small enough to allow the two-dimensional instability to occur, but is the system two-dimensional enough for the quantum Hall effect? The roles of incommensurability and dimensionality need further investigation.

Paul Chaikin is Professor of Physics at the University of Pennsylvania, Philadelphia 19104. and is a Research Associate at the Exxon Research and Engineering Co., Annadale, New Jersey 08801, USA. 